\title{
Effects of a DNA Demethylating Agent - 5-Azacytidine - on Testicular Morphology During Mouse Embryo Development
}

\author{
By
BULUT Hüseyin Eray ${ }^{1)}$, ÖZDEMIR Öztürk ${ }^{2)}$, BAŞIMOGLU-KOCA Yücel ${ }^{3)}$, KORKMAZ Mehmet ${ }^{4)}$ and ATALAY Atilla ${ }^{5)}$

\footnotetext{
1) Department of Histology - Embryology, Faculty of Medicine, Cumhuriyet University, Sivas, Turkey ${ }^{2)}$ Department of Medical Biology and Genetics, Faculty of Medicine, Cumhuriyet University, Sivas, Turkey

3) Department of Biology, Faculty of Science and Arts, Celal Bayar University, Manisa, Turkey

4) Department of Medical Biology, Health High School, The University of Balikesir, Balikesir, Turkey

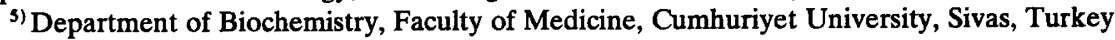

- Received for Publication, November 27, 1998 -

Key Works: 5-azacytidine, Hypomethylation, Embryogenesis, Mouse

Summary: DNA methylation is an epigenetical mechanism that plays crucial roles in cellular differentiation and tissue development in embryogenesis. The aim of the present study was to determine the effects of a demethylating agent, 5 -azacytidine, on testicular development during embryonal life in mouse.

Ten pregnant mice were administered 5-azacytidine (5-azaC) (i.p $2 \mathrm{mg} / \mathrm{kg}$ of agent dissolved in $0.1 \mathrm{mg} / \mathrm{ml}$ PBS) during 8th (Group 1), 11th (Group 2), 14th (Group 3) and 18th (Group 4) days of pregnancy periods and male siblings of these animals were obtained (experimental groups) whereas the control group animals received no treatment and siblings of this group were also obtained. Testicular tissues from all groups were taken 20 days after birth and examined at the light and electron microscopical levels.

All pregnancies were terminated in Group 1 animals, therefore no observations could be done in this group. While Group 2 and 3 siblings showed distinctive kongenital abnormalities such as; anancephaly, growth failure, cleft palate, extremity abnormalities, supernumerary ribs and whirled shaped-tails, no such abnormalities were observed in Group 4 when compared to the control group. Microscopical examination of testicular tissues in groups 2 and 3 demonstrated cellular disintegration of spermatocytes in seminiferous tubules. In addition, cytoplasmic vacuoles and thickening of the basement membrane were also evident in both groups 2 and 3. Apoptotic-like cells were seen especially in group 2 and rarely in group 3. There were no structural alterations in group 4 animals, except a decreased number of spermatocytes in seminiferous tubules when compared to the control group, possibly indicating the completion of embryogenesis in this group.

In conclusion, it could be suggested that the demethylating agent 5-azacytidine may trigger an unknown gene reactivation during early embryogenesis possibly affecting the cell and tissue differentiation in developing mammalian embryos.

The methylation of DNA is used by a wide range of prokaryotes and eukaryotes for the tagging of DNA sequences. It plays crucial roles in both cell types as part of a range of main epigenetic processes, including DNA replication and repair, $\mathrm{X}$-chromosome inactivation, genetic imprinting, apoptosis, regulation of gene expression and restriction/modification systems (Razin and Riggs, 1980; Ehrlich et al., 1981; Ramsahoye et al., 1997; Jost and Bruhat, 1997). The term "DNA methylation" is used to describe a post - replicative modification, in which a DNA residue acquires a covalently bound methyl group. DNA methyltransferases catalyse the transfer of a methyl group from S-adenosylmethionine (SAM) to the position 5 of cytosine in specific DNA sequences. Most, but not all $\mathrm{CpG}$ dinucleotides are methylated in mammalian cells in this manner (Bird, 1980). The methylated base cytosine in $\mathrm{CpG}$ dinucleotides is called 5-methylcytosine (5-mC) or epicytosine. The $5-\mathrm{mC}$ is found in mammalian DNA in the context of the palindromic sequence, $5^{\prime}-\mathrm{CpG}-3^{\prime}$ and called CpG islands (Laird, 1996). It is estimated that $\mathrm{CpG}$ islands are associated with about half of all 
mammalian genes (Antequera and Bird, 1993). Methylation cytosine residues have been found in sequence configurations other than $\mathrm{CpG}$, including CpNpG (Clark et al., 1995). Although it is not known how tissue-specific methylation patterns are established in mammals, it appears that the de novo methylation occurs predominantly in the early embryo (Turker and Bestor, 1997). The precise pattern of methylated cytosine residues varies according to the cell type and developmental stage. Interestingly, imprinted genes were unable to restore their allele - specific methyl patterns until they passed through gametogenesis (Brandeis et al., 1993). However, many mutagens are also known to decrease DNA methylation, another factor known to be important in the regulation of the gene expression. 5-aza-cytidine and 5-aza-2'-deoxycytidine - both cytidine analoges - are known as a potent inhibitors of DNA methylation. These carry a nitrogen atom instead of a carbon atom at the position 5 of the pyrimidine ring. Unlike cytosine, these modified bases cannot accept a methyl group once they are incorporated into DNA (Jhones and Taylor, 1980; Taylor, 1993). 5-aza-C and 5-aza-2'-dC are also inhibited the methylation of newly synthesized DNA, producing inheritable changes in the methylation patterns (Gabbara and Bhagwat, 1995).

It is well known that the cells taking place in the zygote and also in the adult of an eukaryotic organism have the same genomic content, however, the functions and the morphology of these cells become different following the developmental stages of the embryonal life. This is possibly done by the reactivation of stage - specific genes which are triggered by DNA hypomethylation. The above mentioned gene regulation is a sequential mechanism taking place in the zygotic stage throughout the adult life.

The above listed functions of DNA methylation in eukaryotes especially in mammals may play important roles in cell - tissue development during embryogenesis. Therefore present study was designed in mouse testis in order to evaluate the structural effects of demethylating agent, 5-azacytidine (5-azaC), by changing the regular methylation patterns of testicular development.

\section{Materials and Methods}

\section{Animals \\ Animals used in the present study were non - transgenic and were fed in optimal laboratory conditions. Ten female and 2 male Swiss webster albino mice, obtained from The Experimental Ani-}

mal Laboratory of Cumhuriyet University, Sivas Turkey, were housed together overnight. The following morning, the presence of vaginal plugs in female mice confirmed mating and was accepted the first day of pregnancy. Pregnant mice were divided into 5 groups ( 1 control and 4 experimental groups) and demethylating agent was administered to the experimental groups intra-peritoneally.

\section{Experimental Design}

5-azaC was obtained from Sigma Ltd. and stored in $-70^{\circ} \mathrm{C}$. Experimental group pregnant mice were administered $2 \mathrm{mg} / \mathrm{kg} 5$-azaC dissolved in $0.1 \mathrm{mg} / \mathrm{ml}$ cold PBS by intraperitoneal injection on fetal days of $8,11,14$ and 18. The control group pregnant animals received no agent administration. Following 20 days after birth, male siblings of all groups, each of which consisting of 3 animals were sacrificed and the testicular tissues were obtained.

\section{Microscopy}

Mouse testicular tissues were analysed by light and electron microscopy in the present study. For light microscopy, tissue samples were fixed in $10 \%$ neutral formaline, dehydrated in increased concentrations of ethanol and embedded in paraffin blocks. $4-5 \mu \mathrm{m}$ thick sections were obtained and stained with haematoxylene - eosin. Sections were evaluated under a Jenamed 2 (Carl Zeiss Jena, Germany) light microscope and appropriate field of views were photographed.

Tissue samples for electron microscopy were fixed with $2.5 \%$ glutaraldehyde in $0.1 \mathrm{M}$ phosphate buffer ( $\mathrm{pH}$ 7.4) for 2-4 hours. Tissues were rinsed with buffer, post-fixed in $1 \%$ aqueous osmium tetroxide for 2 hours, dehydrated in ethanol, and embedded in Araldite CY 212. Semithin tissue sections were stained with toluidin blue and evaluated at the light microscopical level whereas the ultra-thin sections were double stained with uranyl acetatelead citrate, and observations were done at the ultrastructural level using a Jeol $100 \mathrm{C}$ (Jeol, Japan) electron microscope.

\section{Results}

\section{Control Group}

Light microscopical observations of mouse testicular morphology showed spermatogonia A and B with their dark stained nuclei. Spermatocyte and spermatid series were also seen along with mature spermia filling the tubular lumen. In addition, interstitial tissue contained myoid cells, fibroblasts and Leydig cells (Fig. 1). 


\section{Group 1}

All pregnancies were terminated on day 8 , thus no evaluations could be done morphologically and microscopically in this group.

\section{Group 2 and 3}

Similar findings were observed in these groups. Experimental group mice exposed to the agent on days 11 and 14 (group 2 and 3 respectively) of the intrauterinal life showed distinctive congenital abnormalities such as extremity abnormalities, supernumerary ribs, anancephaly, growth failure, cleft palate and whirl - shaped tails. Light microscopical examination of these groups demonstrated irregularly arranged spermatocyte series in the seminiferous tubuli where there were no mature spermia found in the lumen (Fig. 2). Transmission electron microscopy revealed segmentation and condensation of nuclear chromatin, cell body shrinkage and cytoplasmic vacuolization especially in group 2 testis (Figs. 3, 4). These pathomorphological changes are typical of apoptosis (Fig. 4). These groups also showed thickening of the basement membrane of seminiferous tubules (Fig. 3).

\section{Group 4}

Experimental group mice received the agent administration on day 18 of intrauterinal life demonstrated no external morphological changes. On the other hand, microscopical examination showed testicular features similar to those seen in the control group such as spermatogonia with dark stained nuclei (Fig. 5). Although the cellular features were similar to those seen in the control group, transmission electron microscopical examination of this group revealed a slightly increased vacuolization in the spermatocyte cytoplasm (Fig. 6).

\section{Discussion}

DNA methylation, a postreplicative mechanism, plays a crucial role in gene regulation and tissue differentiation in mammalian embryogenesis. $\mathrm{Hy}-$ pomethylation, performed during this period may cause the reactivation of the stage - specific inactive gene or gene groups resulting with the several abnormalities in different tissues and organs via the alteration of normal molecular lock mechanism of the species - specific methylation patterns (Siegfried and Cedar, 1997). Cytidine analogues (5-aza cytidine and 5-aza-2'-deoxycytidine) are known both potent inhibitors of DNA methylation. Their substitution for cytidine in replicating DNA leads to hypo or demethylation of DNA (Jones et al., 1982; Özdemir and Hornby, 1998). Although it is known how tissue- specific methylation patterns are established in mammals, it appears that extensive de novo methylation occurs predominantly in the early embryo, the methylation patterns are then subsequently inherited via the maintenance of methylation which occurs throughout embryogenesis. In the present study, in order to detect the effects of 5-azaC (a cytidine analogue and anti tumour drug) on testicular morphology, pregnant Swiss webster albino mice were injected with one dose of the agent intraperitoneally on 8th, 11th, 14 th and 18th days of gestation.

The very early stages of embryo development (day 8 of gestation) could not be examined in the present study since those embryos were miscarried possibly by the unknown effects of the agent. $\mathrm{Li}$ and co-workers (1992) suggested the embryonic lethality following the targeted mutation of DNA methyltransferase gene. During the early embryo development the present study found distinctive congenital anomalies such as supernumerary ribs, cleft palate, anancephaly, extremity abnormalities, growth failure and whirl-shaped tail during early embryonic development (11th day of gestation) following 5-azaC administration. Branch et al. (1996) reported similar findings following a different type of cytidine analogue, 5-aza-2'-deoxycytidine, indicating that those base analogues have teratogenic effects on embryo development.

The microscopical examination of testicular tissue revealed distinctive structural changes following 5-azaC administration especially on gestational days 11 and 14 . Those were vacuolization and myelinization in spermatocyte cytoplasm and basement membrane thickening in the seminiferous tubuli. Those structural alterations could be due to the increased protein synthesis in this tissue under the effect of demethylating agent. This possible mechanism of this increased protein synthesis might be the reactivation of functional and/or non-functional genes by the substitution of cytidine with 5azaC, a cytidine analogue, in $\mathrm{CpG}$ dinucleotides. Therefore DNA methyltransferase enzyme could not transfer the methyl groups to this substituted base analogue resulting with the hypomethylation of that regions of DNA. It could also be speculated that this hypomethylation caused by the agent may exert its effect through changing the regular stage specific genomic imprinting mechanism during the embryo development as suggested by Latham (1995).

Transmission electron microscopy revealed segmentation, condensation of nuclear chromatin, and cytoplasmic vacuolization in spermatocytes especially on days 11 and 14 of gestation. These pathomorphological changes were typical of apoptosis 
indicating the effect of 5-azaC on proliferating cells whereas it had no effect after the completion of proliferative stage on day 18 of development. The agent administered on day 18 of development affected slightly the testicular structure when compared to the early stages. The reason for that situation might be due to the resistance of those terminally differentiated cells to the agent.

It has been reported that a number of antitumor drugs, including cisplatin, may exert their toxicity by apoptosis (Hossain et al., 1997a). They demonstrated that 5-azacytidine administered to the dam induced apoptosis in the brains of fetal mice. They also suggested that a cAMP sensitive mechanism is involved in 5-azacytidine induced apoptosis and cell death. Hsiao et al. (1996) declared that rat 6/c-myc cell transfomants induced by 5-azaC lost c-myc induced apoptotic cell death, whereas MnSO4induced transformant cells showed a higher degree of apoptosis than the parental rat $6 / \mathrm{c}$-myc cells. Hossain et al. (1997b) found that 5-azaC, a cytidine analogue, produced apoptosis of fetal developing neuronal cells the day after injection of the agent into dams by intraperitoneal route at the 11 days of gestation. In addition, Murakami and co-workers (1995) suggested that the apoptosis caused by 5azaC in human promyelocytic leukaemic $\mathrm{HL}-60$ cells was occurred especially in $G_{1}$ stage of the cell cycle

The findings of the present study about the presence of apoptotic mechanism in spermatocytes following 5-azaC administration was consistent with previous reports, although they did not performed their studies on testes.

In conclusion, 5-azaC could be a potential teratogenic agent when administered intraperitoneally during mammalian embryo development. The agent may alter the methyl patterns of developing testicular cells. This suggestion was supported by the findings of the present study. Those were testicular morphological changes in mice during the early, mid- and late embryonal stages following the intraperitoneal injection of 5-azaC. The present findings could also indicate the importance of the methylation mechanism during embryo development.

\section{Acknowledgement}

Authors thank to "The State Planning Organization of TURKEY" (DPT) for funding this study.

\section{References}

1) Antequera $\mathrm{F}$ and Bird A. Number of $\mathrm{CpG}$ islands and genes in human and mouse. Proc Natl Sci 1993; 90:1199511999.

2) Bird AP. DNA methylation and the frequency of $\mathrm{CpG}$ in animal DNA. Nucleic Acids Sci Res 1980; 8:1499-1504.

3) Branch S, Francis BM, Brownie CF and Chernoff N. Teratogenic effects of the demethylating agent 5-aza-2-deoxycytidine in the Swiss webster mouse. Toxicology 1996; 112(1):37-43.

4) Brandeis $M$, Ariel $M$ and Cedar $H$. Dynamics of DNA methylation during development. Bio Assays 1993; 45:709713.

5) Clark SJ, Harrison J and Frommer M. CpNpG methylation in mammalian cells. Nat Genet 1995; 10:20-27.

6) Ehrlich $M$, Richard $Y$ and Wang $H$. 5-methylsitosine in eukaryotic DNA. Sci 1981; 212:1350-1357.

7) Gabbara $S$ and Bhagwat AS. The mechanism of inhibition of DNA (cytosine-5-) methyltransferases by 5-azacytosine is likely to involve methyl transfer to the inhibitor. Biochem J 1995; 307:87-92.

8) Hossain MM, Takashima A, Nakayama $H$ and Doi K. 5Azacytidine induced toxicity in PC12 cells by apoptosis. Exp Toxicol Pathol 1997a; 49(3-4):201-206.

9) Hossain MM, Nakayama $\mathrm{H}$ and Goto $\mathrm{N}$. In vitro induction of apoptosis of developing brain cells by 5 -azacytidine. Int $\mathrm{J}$ Dev Neurosci 1997b; 14(1):11-17.

10) Hsiao WL, Mendosa G, Kothari NH and Fan H. Comparison of transformation by manganese sulphate and 5-azacytidine in rat 6 cells overexpressing the c-myc oncogene. Carcinogenesis 1996; 17(12):2771-2777.

11) Jones PA and Taylor SM. Cellular differentiation, cytidine analogues and DNA methylation. Cell 1980; 20:85-93.

12) Jones PA, Taylor SM, Mohandas T and Shapiro LJ. Cell cycle-specific reactivation of an inactive X-chromosome locus by 5-azadeoxycytidine. Proc Natl Acad Sci USA 1982; 79:1215-1219.

13) Jost JP and Bruhat A. The formation of DNA methylation patterns and the silencing of genes. Prog Nucleic Acid Res Mol Biol 1997; 57:217-248.

14) Laird PW. The role of DNA methylation in cancer genetics and epigenetics. Annu Rev Genet 1996; 30:441-464.

15) Latham KE. Stage-specific and cell type-specific aspects of genomic imprinting effects in mammals. Differentiation 1995; 59(5):269-282.

16) Li E, Bestor TH and Jaenisch R. Targeted mutation of the DNA methyltransferase gene results in embryonic lethality. Cell 1992; 69:915-926.

17) Murakami T, Li X, Gong J, Bhatia U, Traganos $F$ and Darzynkiewicz Z. Induction of apoptosis by 5-azacytidine: drug concentration - dependent differences in cell cycle specificity. Cancer Res 1995; 55(14):3093-3098.

18) Özdemir Ö and Hornby DP. In vivo DNA methylation of E. coli $\mathrm{DH} \alpha 5$ and Top $10 \mathrm{~F}^{\prime}$ strains by cytosine-5 methyltransferase M.Msp1. Tr J Biol 1998; 22(2):143-151.

19) Ramsahoye BD, Davies CS and Mills KI. DNA methylation: biology and significance. Blood Rev 1997; 10(4):249 261.

20) Razin A and Riggs AD. DNA methylation and gene function Sci 1980; 210:604-610.

21) Siegfried $Z$ and Cedar H. DNA methylation: a molecular lock. Curr Biol 1997; 7(5):R305-307.

22) Taylor SM. 5-aza-2'-deoxycytidine: cell differentiation and DNA methylation. Leukaemia. Suppl. Monograph 1993; 7:3-8.

23) Turker MS and Bestor TH. Formation of methylation patterns in the mammalian genome. Mutat Res 1997; 386(2):119-130. 
Plate I
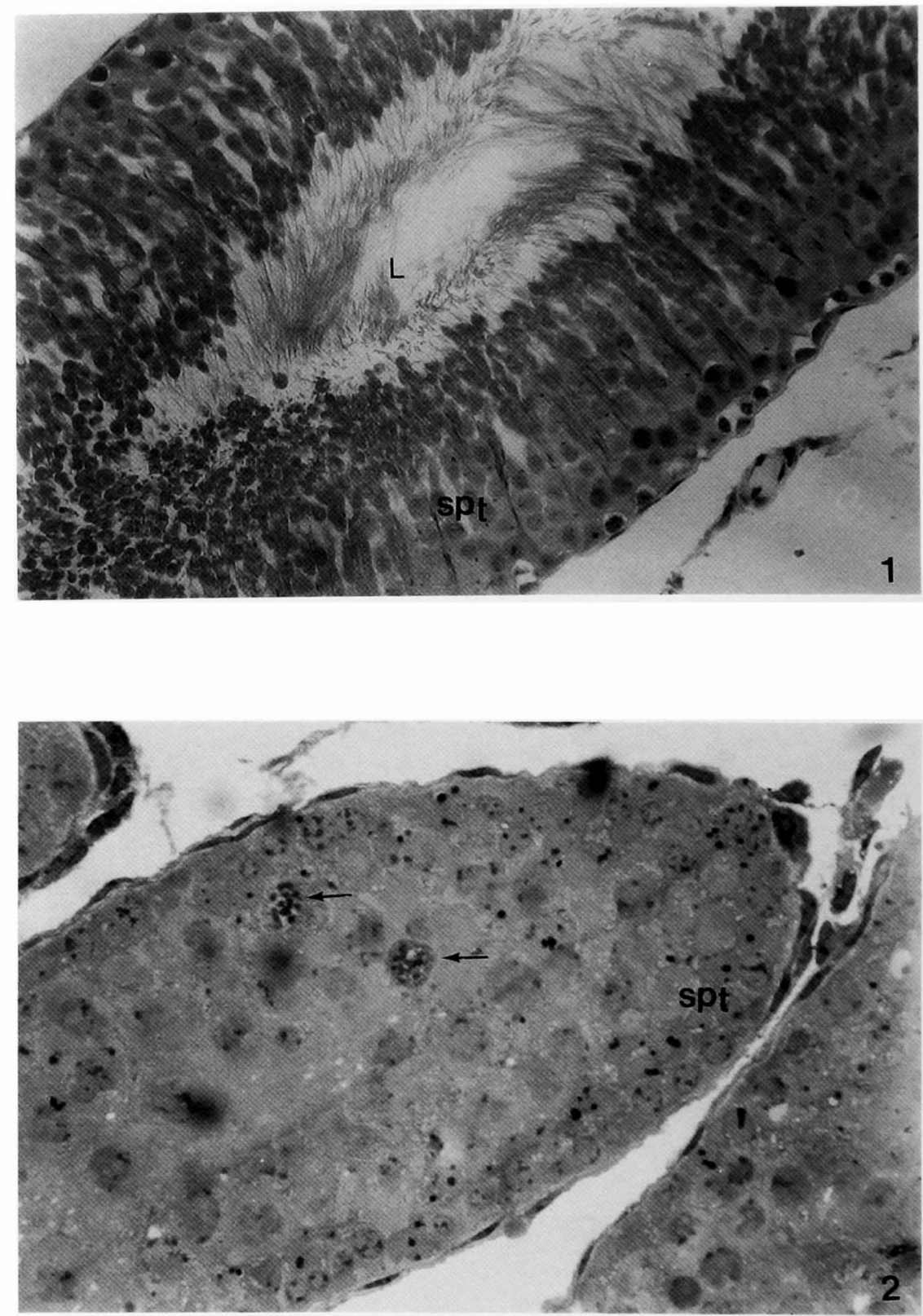

\section{Explanation of Figures}

\section{Plate I}

Fig. 1. Shows the control group testicular structure at the light microscopical level. L: lumen, spt: spermatocyte. $\times 400$, toluidin blue - acid fuchsin.

Fig. 2. Shows the Group 2 and 3 testicular morphology containing apoptotic - like cells $(\rightarrow)$ at the light microscopical level. spt: spermatocyte. $\times 400$, toluidin blue. 
Plate II
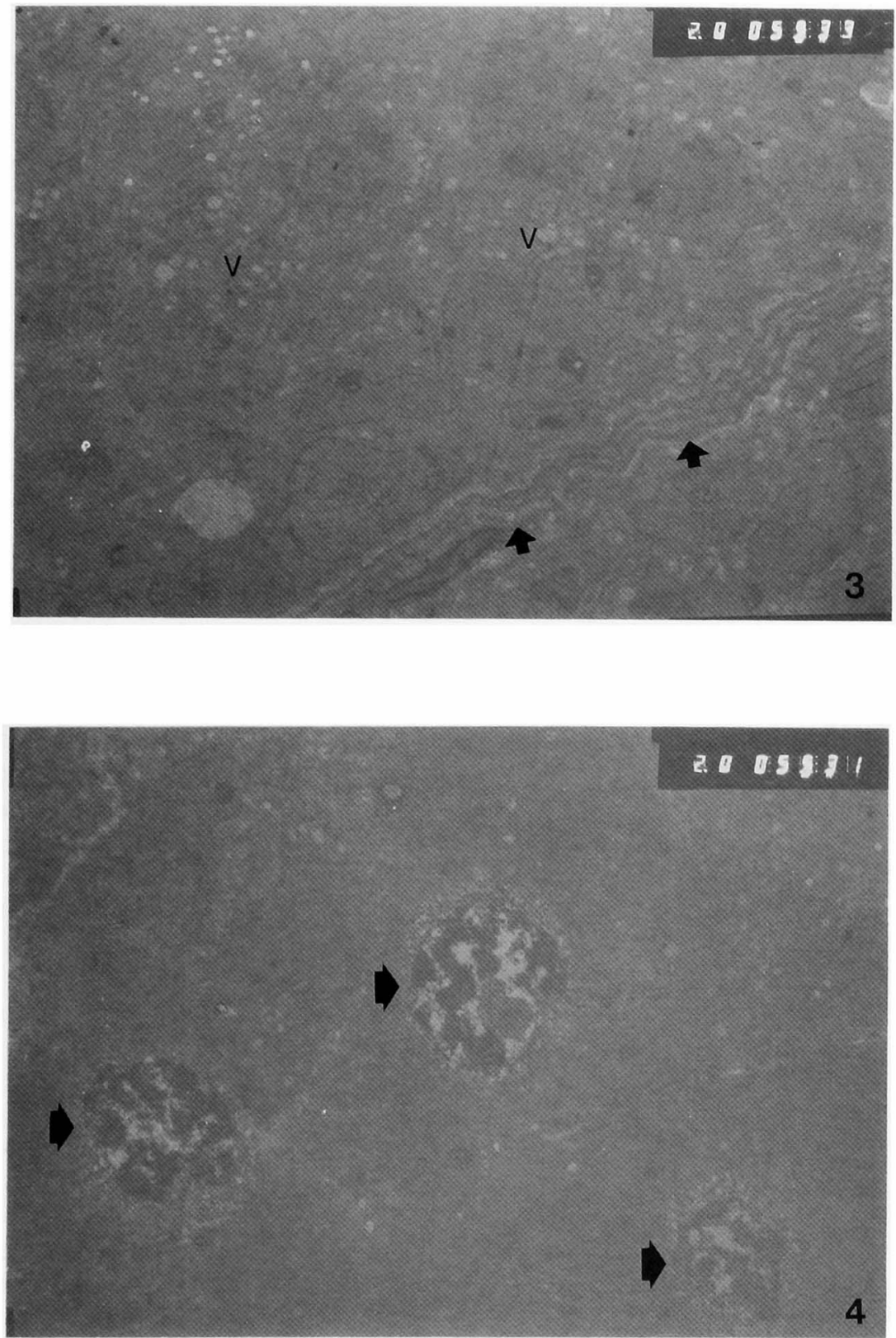

\section{Plate II}

Fig. 3. Transmission electron microscopical examination of group 2 and 3 testicular ultrastructure demonstrates increased basement membrane thickness $(\rightarrow)$ and abundant cytoplasmic vacuolization (V) in the tubular cells. $\times 7000$.

Fig. 4. Shows the ultrastructural appearance of apoptotic cells $(\Rightarrow)$ in Group 2 along with nuclear compartmentalization, segmentation and condensation which are the features of mid-level apoptosis. $\times 7000$. 

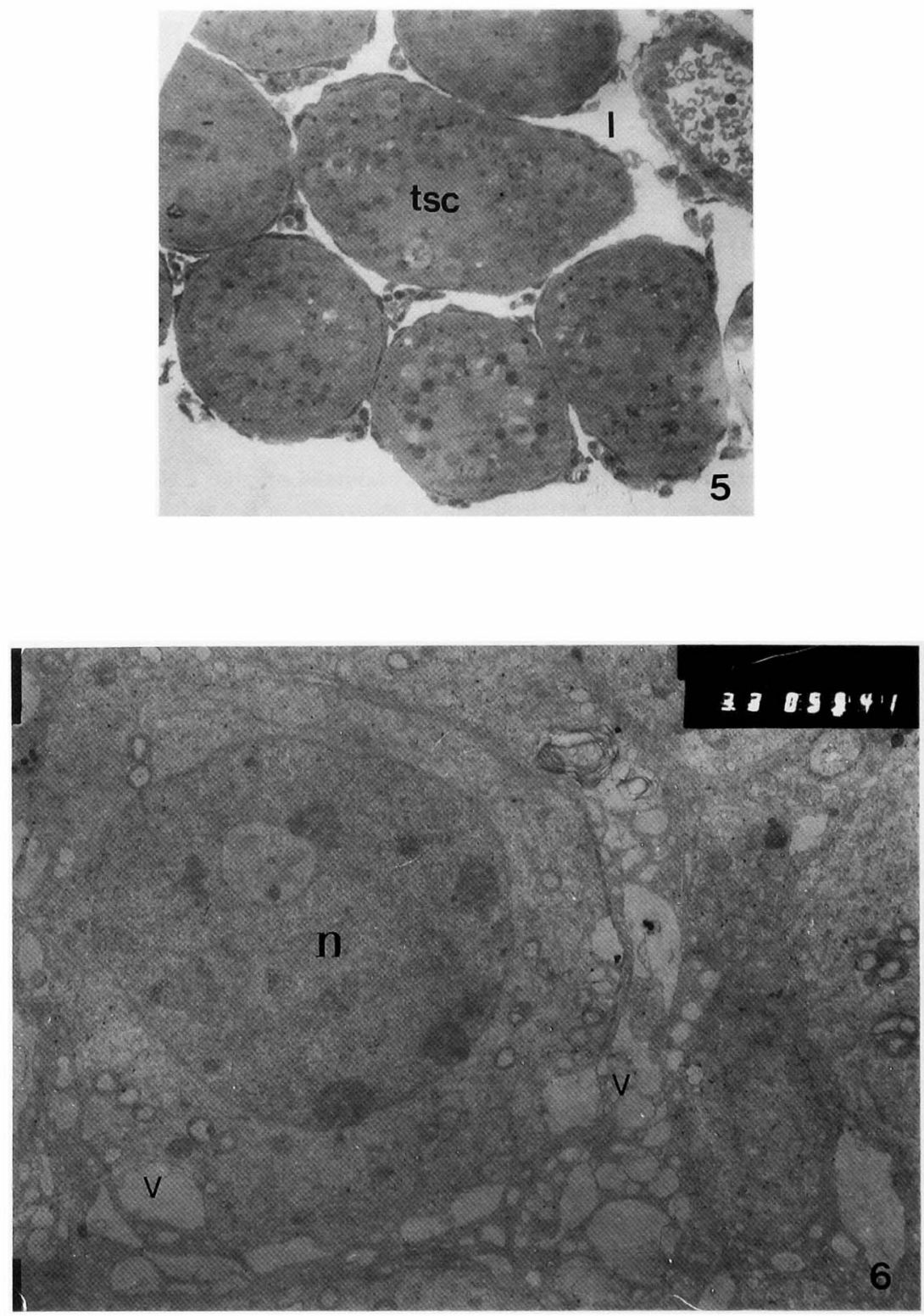

Plate III

Fig. 5. Demonstrates the light microscopical appearance of Group 4 testicular features which are normal as seen in the control group. tsc: seminiferous tubuli, I: interstitium. $\times 200$, toluidin blue - acid fuchsin.

Fig. 6. Transmission electron microscopy of Group 4 contains cellular structure similar to those seen in the control group with a slightly increased cytoplasmic vacuolization (V). n: nucleus. $\times 11000$. 